\title{
Identifying Group-Wise Consistent White Matter Landmarks via Novel Fiber Shape Descriptor
}

\author{
Hanbo Chen ${ }^{1}$, Tuo Zhang ${ }^{1,2}$, and Tianming Liu ${ }^{1}$ \\ ${ }^{1}$ Department of Computer Science and Bioimaging Research Center, \\ The University of Georgia, Athens, GA, USA \\ ${ }^{2}$ School of Automation, Northwestern Polytechnical University, Xi'an, China
}

\begin{abstract}
Identification of common and corresponding white matter (WM) regions of interest (ROI) across human brains has attracted growing interest because it not only facilitates comparison among individuals and populations, but also enables the assessment of structural/functional connectivity in populations. However, due to the complexity and variability of the WM structure and a lack of effective white matter streamline descriptors, establishing accurate correspondences of WM ROIs across individuals and populations has been a challenging open problem. In this paper, a novel fiber shape descriptor which can facilitate quantitative measurement of fiber bundle profile including connection complexity and similarity has been proposed. A novel framework was then developed using the descriptor to identify group-wise consistent connection hubs in WM regions as landmarks. 12 group-wise consistent WM landmarks have been identified in our experiment. These WM landmarks are found highly reproducible across individuals and accurately predictable on new individual subjects by our fiber shape descriptor. Therefore, these landmarks, as well as proposed fiber shape descriptor has shown great potential to human brain mapping.
\end{abstract}

Keywords: White Matter Landmark, DTI, Group-wise Consistency, Fiber Shape Descriptor.

\section{$1 \quad$ Introduction}

Identification of common and corresponding white matter (WM) regions of interest (ROIs) across human brains has attracted growing interest not only in that it facilitates comparison among individuals and populations, but also because it makes it possible to assess structural/functional connectivity in populations [1]. One mainstream of previous methods developed in the field so far largely relies on building white matter atlases via registration methods [2,3] to establish correspondence across subjects. Alternatively, voxel based or fiber tract based features have been newly applied to identify WM ROIs. For instance, in [4], FOD (fiber orientation distribution) has been applied to identify pathologies. In [5], group-wise shape analysis based on fiber tracts has been performed to study WM. In [6], an effective fiber bundle shape descriptor called trace-map has been developed. Base on the trace-map feature [6], a map of discrete cortical landmarks named DICCCOL [7] that possess group-wise consistent white matter fiber connection patterns across individuals has been identified. 
However, identifying reliable WM landmarks is still a challenging open problem due to the complexity and variability of the brain structure and a lack of effective white matter streamline descriptors [1]. For instance, the accuracy and reliability of registration based method are limited due to the substantial variability in brain anatomy and structure between individuals. As for most voxel based methods, they are based on local information and have difficulty in establishing between-subject correspondences. In [7], the authors successfully solved the above mentioned issues by introducing an effective fiber bundle shape descriptor of trace map [6]. However, since those identified DICCCOL landmarks locate on cortical surface, the remarkable cortical folding pattern variation may be a major barrier to further improvement.

Motivated by the achievements and the challenges in [6], we developed a novel shape descriptor to characterize the connection patterns of a fiber bundle. Instead of focusing on the shape of streamline fibers as in [6], our descriptor centers on the global connection pattern of fibers. In particular, our proposed descriptor is based on probability density which enables the measurement of directional statistic features. Based on this descriptor, an effective searching/optimization framework is designed to identify WM landmarks that: 1) are highly connected hubs in the brain; 2) are reproducible across individuals. The reason we aim to identify hubs as landmarks lies in that the human brain networks have been shown to be a small-world network [8]. In such network, hubs are more robust, consistent, and could be used to identify subnodes. Thus, by identifying hubs in WM, those landmarks can be potentially used as initial points to establish correspondences across individuals, which will bring great potential to the study of human brain mapping, such as WM landmark-guided image registration. In comparison to existing model-driven WM landmark identification methods [2, 3], the major novelty of our work is that it is data-driven and thus can better handle the complexity and variability of the WM architecture.

\section{Methods}

In this paper, brain ROI is defined as a sphere in the space. To identify WM landmarks with abovementioned properties from ROIs, first, we borrowed the idea from [6] and defined a novel spherical probabilistic distribution based connection map feature vector to describe the fiber profile (Fig. 1(b)). Then, the connection pattern complexities of ROIs are measured to identify WM landmarks (Fig. 1(c-d)). Finally, the locations of landmarks are iteratively optimized in each subject's own space to increase group-wise consistency of these landmarks' connection profiles (Fig. 1 (e)). The computational pipeline of the proposed framework is summarized in Fig. 1.

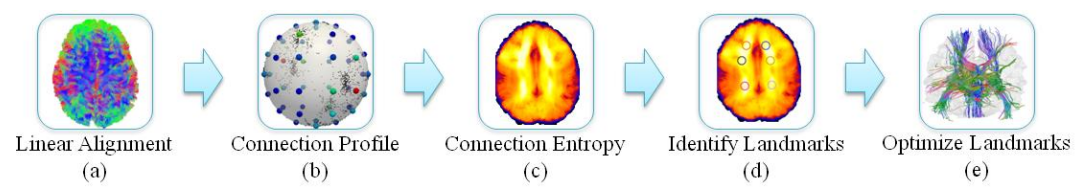

Fig. 1. Illustration of the computational pipeline, consisting of 5 steps 


\subsection{ROI Connection Map}

First, definitions of several key concepts and terms are given.

ROI fiber bundle: An ROI is defined as a sphere with a predefined radius $(5 \mathrm{~mm}$ in this paper). The fiber streamlines passing through this sphere is viewed as the fiber bundle of the corresponding ROI.

Fiber principal orientation: For each fiber, its principal orientation is described by a norm vector $\boldsymbol{v}$ which is the first principal component of the points $\boldsymbol{X}$ along the fiber such that:

$$
\max \left(\sum_{x_{i} \in X}\left(v \cdot\left(x_{i}-\bar{x}\right)\right)^{2}|| v \mid=1\right)
$$

where $\bar{x}$ is the center of the points in $\boldsymbol{X}$.

Fiber connection profile: For a bundle of fibers, by projecting the principal orientation $v$ of each fiber to a unit sphere, the connection profile of this fiber bundle can be represented and interpreted by the points distributed on the sphere. As illustrated in Fig. 2(a-b), the complex fiber bundle connection pattern is mapped to the surface of a sphere without the loss of global information and could be further reduced in dimension via directional statistics method. Notably, as fiber is non-directional connection, both its principal direction $\boldsymbol{v}$ and the opposite direction $\boldsymbol{v} \boldsymbol{v}$ will be projected to the sphere.

Connection map: The probability density of orientation vector on the sphere is applied to describe the connection map of fiber bundles. Specifically, the sphere is subdivided into 48 equal sized pixels as defined in the HEALPix [9]. The number of points within each pixel out of the total number of points is calculated as the probability density. In this way, the fiber connection profile is represented by a connection map with a vector of 48 numbers such that:

$$
\begin{gathered}
P(V)=\left\{P_{1}(V), P_{2}(V), \cdots, P_{48}(V)\right\} \\
P_{k}(V)=\frac{\left\|V \cap R_{k}\right\|}{\|V\|}(k=1 \cdots 48)
\end{gathered}
$$

where $R_{k}$ is the area covered by pixel $k$, and $\|V\|$ is the number of points on the unit sphere. The advantage of this representation is the capability of representing complex fiber bundle connection pattern with a simple one dimensional feature vector without the loss of major information. As shown in Fig. 2, the connection maps are similar for the fiber bundles sharing similar shapes. For the bundles with different shapes, the connection maps would be distinct. Notably, this representation may have difficulty in distinguishing the fiber bundles with similar shapes but connecting in the opposite direction, or the fiber bundles with similar orientations but different lengths (e.g. the anterior thalamic projection V.S. the inferior fronto-occipital and uncinate fasciculus). These issues could potentially be solved by defining fiber connection direction and introducing multiple spherical shells for fiber bundles with different lengths.

Connection entropy: As the HEALPix pixels [9] are the squares evenly distributed on the sphere with equal size, the entropy of orientation vectors $V$ distributed on the sphere could be directly obtained from $\boldsymbol{P}(\boldsymbol{V})$ :

$$
H(V)=\sum_{i=1 \cdots 48}-P_{i}(V) \log _{48} P_{i}(V)
$$


Connection similarity: The similarity between two connection maps is measured by cosine similarity:

$$
S\left(P\left(V_{i}\right), P\left(V_{j}\right)\right)=\frac{P\left(V_{i}\right) \cdot P\left(V_{j}\right)}{\left\|P\left(V_{i}\right)\right\| \cdot\left\|P\left(V_{j}\right)\right\|}
$$

Both connection entropy and similarity are values between 0 and 1. As shown in Fig. 2 , higher connection entropy indicates higher connection pattern complexity, and higher similarity value indicates higher similarity between fiber bundles.

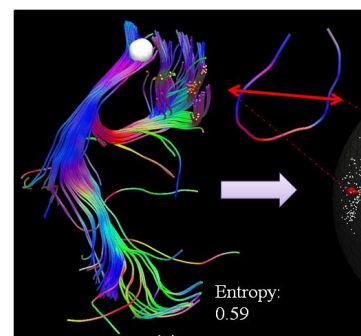

(a)

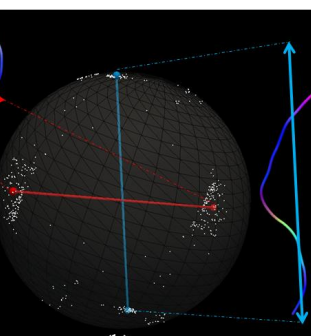

(b)
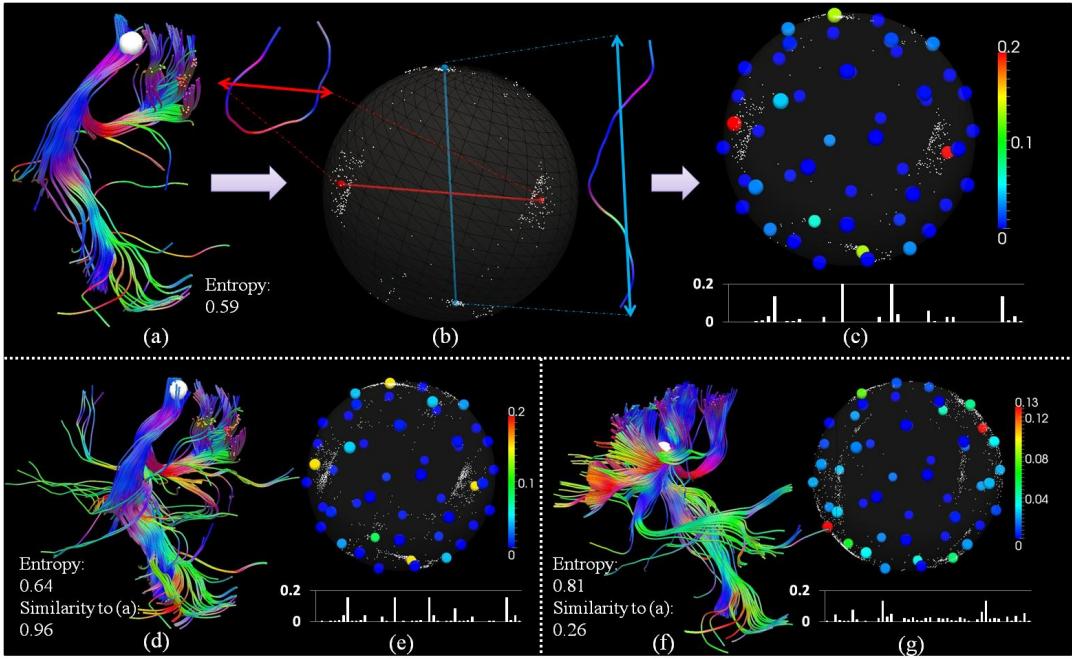

Fig. 2. Illustration of ROI connection profile. (a), (d), (f): DTI-derived fibers connected to an ROI. The ROI is defined by a sphere in space (white bubble). (b) Illustration of projecting fibers' orientations to a sphere to obtain connection profile. The white dots are the projection points of fibers. The red and blue lines indicate the main orientation of the corresponding fibers and their projections on the unit sphere. (c), (e), (g): The probabilistic distributions of connection profiles of the fiber bundles shown on their left. The center of each sampling pixel is shown and color-coded by the probability density. Corresponding connection map feature vector is shown at the bottom by histogram.

\subsection{Identify, Optimize and Predict WM Landmarks}

To identify the group-wise consistent WM landmarks that play the hub roles, we need to find the ROIs that maximize the connection entropy (meaning more diverse connections) and cross-subject connection similarity (meaning group-wise consistency) at the same time. The optimization is thus designed to maximize the energy function (equivalent to minimizing the distance function) described below:

$$
\begin{gathered}
E(X)=\sum_{x_{i} \in X}\left(E_{\text {int }}\left(x_{i}\right)+\lambda E_{\text {ext }}\left(x_{i}\right)\right) \\
E_{\text {int }}\left(x_{i}\right)=\sum_{j=1 \cdots n} H\left(V\left(F\left(x_{i}^{j}\right)\right)\right)
\end{gathered}
$$




$$
E_{e x t}\left(x_{i}, \bar{X}\right)=\sum_{j=1 \cdots n} S\left(V\left(F\left(x_{i}^{j}\right)\right), \overline{V(F(X))}\right)
$$

where $\boldsymbol{X}$ is the set of a landmark in all subjects. $E_{\text {int }}\left(x_{i}\right)$ is the internal energy function of connection entropy. $E_{\text {ext }}\left(x_{i}\right)$ is the external energy function of connection similarity. $\lambda$ is the tradeoff (empirically set to 1 in this paper). $F\left(x_{i}^{j}\right)$ is the fiber bundle passing through the landmark $x_{i}$ of subject $j$.

The landmark searching framework follows the pipeline shown in Fig. 1. First, the subjects are initially aligned to the same space by linear registration (FSL FLIRT [10]). Then, taking each voxel in the space as the center of an ROI, the connection map entropy of fiber bundles passing through each ROI is computed for each subject. By averaging these connection entropy images, a group-wise connection entropy map is obtained (Fig. 3(a)). Distinguishable regions with high average connection entropy values in this map are visually identified as the initial landmarks. Due to the individual variability and misalignment, the fiber profiles of these initial landmarks could be different. To solve this problem, the landmarks' locations are optimized iteratively with random walks in each subject to maximize the energy function in Eq. (6) as described in Algorithm 1. After optimization, the converged landmarks are then used as template for prediction on new individual brains. The prediction process used a similar framework as optimization. First, the brain of new subject with DTI data is registered to the template space. Then, the space around the initial location of each landmark is searched for the point that maximizes external energy $E_{\text {ext }}\left(x^{*}, \bar{X}_{\text {template }}\right)$ defined in Eq. (8) to guarantee the similarity with template.

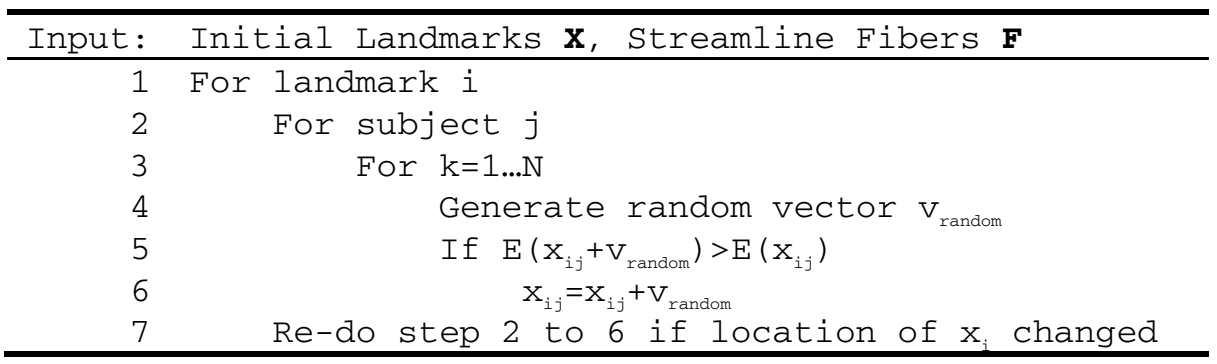

\section{Algorithm 1.}

\section{Experiment Results}

Two sets of data are applied. One is acquired from 18 healthy young adults who are equally distributed as training subjects and prediction testing subjects. The parameters are: matrix size $128 \times 128$, 60 slices, image resolution $2 \times 2 \times 2 \mathrm{~mm}^{3}$ isotropic, $\mathrm{TR}=15 \mathrm{~s}$, $\mathrm{ASSET}=2,3 \mathrm{~B} 0$ images, 30 optimized gradient directions, b-value $=1000$. Another dataset publicly released by Human Connectome Project (HCP)[11] contains DTI data of 64 healthy subjects. This dataset is applied to test prediction of trained landmarks. The DTI data preprocessing was performed via FSL [10] which includes eddy current correction, skull removal, computing FA image, tissue segmentation, and linear registration. Fiber tracking was performed via MedINRIA [12] using streamline model and then registered to the same space. 


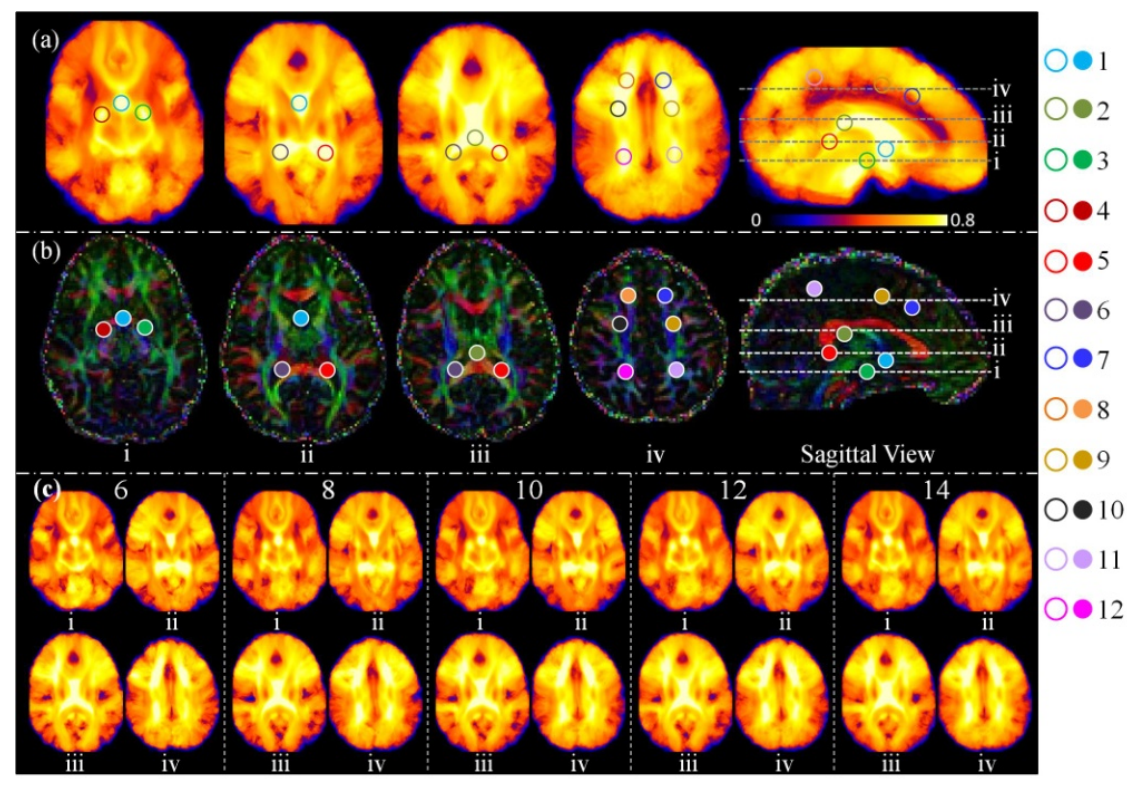

Fig. 3. Visualization of average entropy map and the location of 12 landmarks. The location of each landmark is indicated by colored ring/bubble. The location of each axial plan is illustrated by dash line in the sagittal view on the right. (a) Average entropy map of 9 randomly pick subjects. (b) Slices of RGB color-coded principal diffusion tensor direction of template subject volume with corresponding view to (a). (c) Average entropy map of randomly picked subjects with different numbers $(6 / 8 / 10 / 12 / 14)$. The view is the same as (a).

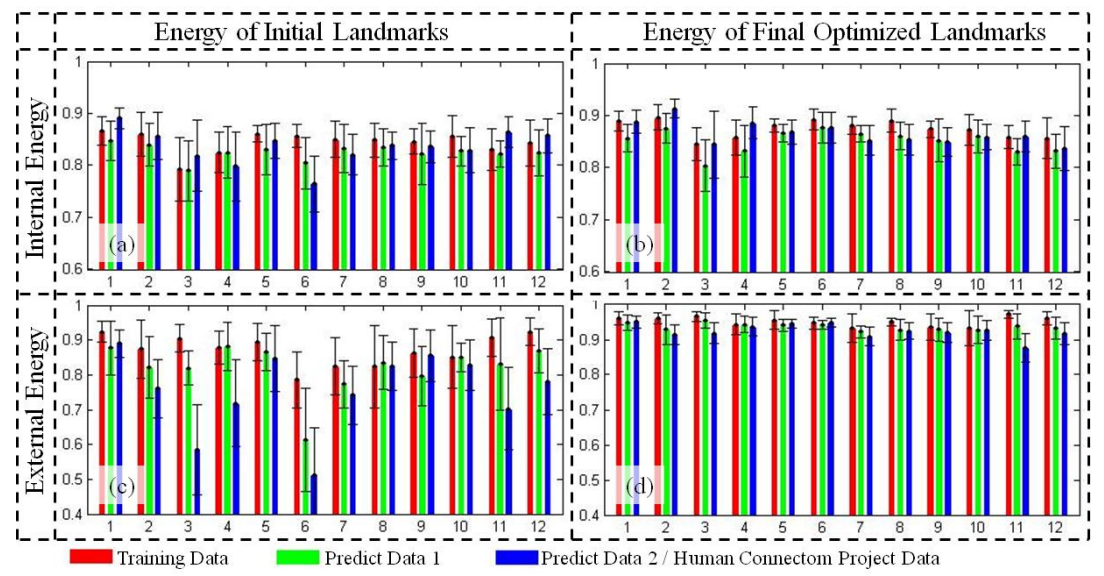

Fig. 4. Average internal and external energy of each landmark in different dataset

After preprocessing, entropy map is computed for randomly picked subjects with different subject numbers $(6 / 8 / 9 / 10 / 12 / 14)$. The result is similar and consistent between different groups with different sizes Fig. 3(a,c). Thus we randomly picked 9 subjects for training. Finally, 12 landmarks with high connection entropy were 
visually identified and then automatically optimized as shown in Fig. 3(a-b). Taking the training subjects as the template, these 12 landmarks are predicted on other 9 subjects as well as 64 subjects from HCP data for validation. The average internal energies and external energies are shown in Fig. 4. For training data, both internal and external energies are relatively high, which indicates that these landmarks are consistent connection hubs in brain WM. For prediction data, compared with initial landmarks (obtained via linear registration), the energies of finally optimized landmarks are much higher, which suggests that these landmarks are very reproducible in new subjects and could be predicted with our proposed framework.

To further examine anatomical meaning of these landmarks, we randomly picked one training subject and one prediction subject to visualize major pathways passing these landmarks (Fig. 5). By observation, all these landmarks locate at the intersection point or the connection concentration regions of major fiber pathways. For instance, landmark 3 and 4 are close to the thalamus of each brain spheres, and the fiber tracts such as thalamic radiations, corticopontine tract and corticalspinal tract concentrate around this region. For landmark 5 and 6, the fiber pathways from different lobes intersect at this region, e.g. corpus callosum, posterior thalamic radiation, superior longitudinal fasciculus, and superior longitudinal fasciculus, and stria terminalis/fornix. The pathways that go through these landmarks will be further examined in our future work.

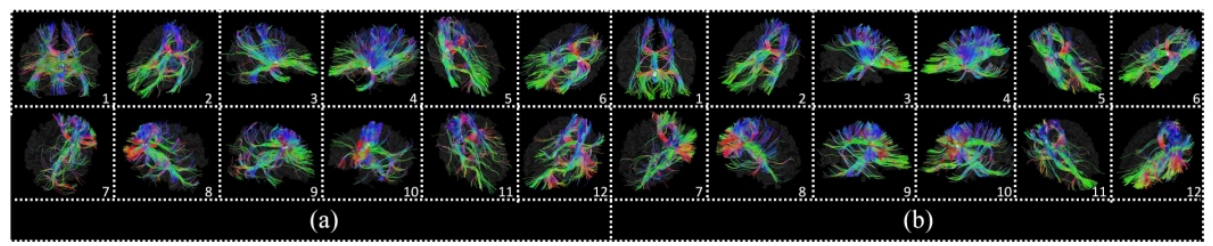

Fig. 5. Visualization of fiber bundle of 12 landmarks of (a) a training subject and (b) a testing subject. The landmarks are represented by white bubbles in each sub-figure. The IDs of the landmarks are listed on the right-bottom corner.

\section{Conclusion and Discussion}

A novel fiber shape descriptor has been proposed to characterize the connection patterns of a fiber bundle. The advantage of this descriptor lies in that: (1) it is based on orientation probability density distribution, thus enabling direct calculation of directional statistic features such as similarity or entropy; (2) it can be efficiently calculated with a decision tree which makes it fast to compute. Our validation experiment suggests that this descriptor can quantitatively measure the similarity and connection complexity of fiber bundles with high accuracy. Based on proposed descriptor, a novel computational framework has been developed to identify and predict landmarks that are group-wise consistent connection hubs in WM region. Finally, 12 landmarks with dense connection and high reproducibility across individuals are identified and validated. The major novelty and contribution of WM landmark discovery framework lies in its data-driven nature that can better handle the complexity and variability of the WM structures, in comparison to existing model-driven methods.

In the future, these WM landmarks will be used as initial points to establish crossindividual correspondences for brain image registration. Also, a hierarchical brain 
connection map will be built based on these WM landmarks to facilitate brain structural/functional connection studies in population. For instance, with each individual, we will first recognize their WM landmarks and then the consistent cortical regions that are connected to these landmarks. Afterward, the sub-connected regions between these consistent cortical regions could be recognized iteratively to populate the landmarks on the cortical brain with consistent connectomes. Finally, it is noted that future applications of the proposed fiber shape descriptor is not limited to the framework introduced. It has broader potential to be applied in fiber shape related clustering problems such as connection based cortical segmentation optimization [13] or diffusion tensor image based functional ROIs prediction [14].

Acknowledgements. Data were provided [in part] by the Human Connectome Project, WU-Minn Consortium (Principal Investigators: David Van Essen and Kamil Ugurbil; 1U54MH091657) funded by the 16 NIH Institutes and Centers that support the NIH Blueprint for Neuroscience Research; and by the McDonnell Center for Systems Neuroscience at Washington University.

\section{References}

1. Derrfuss, J., Mar, R.A.: Lost in localization: the need for a universal coordinate database. NeuroImage 48, 1-7 (2009)

2. Mori, S., et al.: Stereotaxic white matter atlas based on diffusion tensor imaging in an ICBM template. NeuroImage 40, 570-582 (2008)

3. Yap, P.-T., Wu, G., Zhu, H., Lin, W., Shen, D.: TIMER: tensor image morphing for elastic registration. NeuroImage 47, 549-563 (2009)

4. Bloy, L., Ingalhalikar, M., Eavani, H., Roberts, T.P.L., Schultz, R.T., Verma, R.: HARDI based pattern classifiers for the identification of white matter pathologies. In: Fichtinger, G., Martel, A., Peters, T., et al. (eds.) MICCAI 2011, Part II. LNCS, vol. 6892, pp. 234-241. Springer, Heidelberg (2011)

5. O'Donnell, L.J., Westin, C.-F., Golby, A.J.: Tract-based morphometry for white matter group analysis. NeuroImage 45, 832-844 (2009)

6. Zhu, D., Zhang, D., Faraco, C., Li, K., Deng, F., Chen, H., Jiang, X., Guo, L., Miller, L.S., Liu, T.: Discovering dense and consistent landmarks in the brain. In: Székely, G., Hahn, H.K., et al. (eds.) IPMI 2011. LNCS, vol. 6801, pp. 97-110. Springer, Heidelberg (2011)

7. Zhu, D., et al.: DICCCOL: Dense Individualized and Common Connectivity-Based Cortical Landmarks. Cerebral Cortex. (2012)

8. Bullmore, E., Sporns, O.: Complex brain networks: graph theoretical analysis of structural and functional systems. Nature Reviews. Neuroscience 10, 186-198 (2009)

9. Gorski, K.M., et al.: HEALPix: A Framework for High-Resolution Discretization and Fast Analysis of Data Distributed on the Sphere. The Astrophysical Journal 622, 759-771 (2005)

10. Jenkinson, M., et al.: FSL. NeuroImage 62, 782-790 (2012)

11. Van Essen, D.C., et al.: The Human Connectome Project: a data acquisition perspective. NeuroImage 62, 2222-2231 (2012)

12. Toussaint, N., Souplet, J., Fillard, P.: MedINRIA: Medical image navigation and research tool by INRIA. In: MICCAI. pp. 1-8 (2007)

13. Clarkson, M.J., Malone, I.B., Modat, M., Leung, K.K., Ryan, N., Alexander, D.C., Fox, N.C., Ourselin, S.: A framework for using diffusion weighted imaging to improve cortical parcellation. In: Jiang, T., Navab, N., Pluim, J.P.W., Viergever, M.A., et al. (eds.) MICCAI 2010, Part I. LNCS, vol. 6361, pp. 534-541. Springer, Heidelberg (2010)

14. Zhang, T., et al.: Predicting functional cortical ROIs via DTI-derived fiber shape models. Cerebral Cortex. 22, 854-864 (2012) 